\title{
Effects of non-local exchange on core level shifts for gas-phase and adsorbed molecules
}

\author{
M. Van den Bossche, ${ }^{1}$ N. M. Martin, ${ }^{2}$ J. Gustafson, ${ }^{2}$ C. Hakanoglu, ${ }^{3}$ J. F. Weaver, ${ }^{3}$ \\ E. Lundgren, ${ }^{2}$ and $\mathrm{H}$. Grönbeck ${ }^{1}$ \\ ${ }^{1}$ Department of Applied Physics and Competence Centre for Catalysis, Chalmers University of Technology, \\ Göteborg, Sweden \\ ${ }^{2}$ Division of Synchrotron Radiation Research, Lund University, Lund, Sweden \\ ${ }^{3}$ Department of Chemical Engineering, University of Florida, Gainesville, Florida 32611, USA
}

(Received 7 May 2014; accepted 30 June 2014; published online 21 July 2014)

\begin{abstract}
Density functional theory calculations are often used to interpret experimental shifts in core level binding energies. Calculations based on gradient-corrected (GC) exchange-correlation functionals are known to reproduce measured core level shifts (CLS) of isolated molecules and metal surfaces with reasonable accuracy. In the present study, we discuss a series of examples where the shifts calculated within a GC-functional significantly deviate from the experimental values, namely the CLS of $\mathrm{C} 1 s$ in ethyl trifluoroacetate, $\mathrm{Pd} 3 d$ in $\mathrm{PdO}$ and the $\mathrm{O} 1 s$ shift for $\mathrm{CO}$ adsorbed on $\mathrm{PdO}(101)$. The deviations are traced to effects of the electronic self-interaction error with GC-functionals and substantially better agreements between calculated and measured CLS are obtained when a fraction of exact exchange is used in the exchange-correlation functional. (ㅇ 2014 Author(s). All article content, except where otherwise noted, is licensed under a Creative Commons Attribution 3.0 Unported License. [http://dx.doi.org/10.1063/1.4889919]
\end{abstract}

\section{INTRODUCTION}

An important application of first principles calculations is to facilitate the interpretation of different spectroscopic measurements. One example is X-ray photo-emission spectroscopy (XPS) where the combination of high resolution data and calculations frequently are used to characterize adsorbates and determine surface structures. ${ }^{1,2,49}$ The use of XPS for chemical analysis is based on the fact that the core level binding energies are sensitive to changes of the electron density in the valence region. First principles calculations within the Density Functional Theory (DFT) have repeatedly been demonstrated to reproduce experimental core level shifts (CLS) with reasonable accuracy. By use of local or gradient-corrected (GC) functionals, calculated shifts are for many systems rather insensitive to details in the form of the exchange-correlation functional. ${ }^{3-5}$ This is related to error cancellations based on the localized nature of the core states. 6,7

CLS are often discussed in terms of initial and final state contributions. The initial state effect refers to the binding energy of the core electron before the photo-emission process and is a measure of the chemical environment of the core state. The final state effects arise from electronic screening of the hole in the core region when the photoelectron is removed. As the lifetime of the core hole generally is long with respect to the photo-emission process, experiments measure the binding energies including final state effects. ${ }^{8}$ The CLS is usually evaluated as the difference in ground state total energy of the system with a core hole in a reference atom and with a core hole in the atom of interest. This is a method that assumes complete screening of the core hole. The approach is widely used and the good agree- ment with experiments have been demonstrated in several cases. ${ }^{9-15}$

Although GC exchange-correlation functionals predict CLS with reasonable accuracy, such functionals have an intrinsic self-interaction problem, ${ }^{16}$ i.e., a spurious interaction of each electron with itself that arises due to incomplete cancellation between these terms in the direct Coulomb and exchange integrals. The self-interaction error (SIE) is manifested in many electronic properties, where the underestimation of the band gap of metal oxides is one well-known example. A common scheme to correct for the SIE is to add a part of Fock exchange in, so-called, hybrid functionals. Reduction of the SIE leads to a higher degree of electron localization which for some systems could be accomplished with the DFT $+\mathrm{U}$ methodology. ${ }^{17}$ The effects of hybrid functionals on CLS have recently been discussed in the literature. ${ }^{18,19}$ Binder et al. ${ }^{18}$ investigated a set of germanium containing molecules and showed that whereas the Ge $3 d$ CLS evaluated within a GC-functional had a root mean square deviation (RMSD) of $0.19 \mathrm{eV}$, a functional with a fraction of exact exchange yielded a better quantitative agreement with experiment (RMSD of $0.09 \mathrm{eV})$. Also for water adsorbed on $\mathrm{MgO}(100)$, the effect of a hybrid functional on the O $1 s$ CLS has been calculated to be in the range of $\sim 0.2 \mathrm{eV} .^{19}$

In the present contribution, we discuss CLS of PdO and $\mathrm{CO}$ adsorbed on $\mathrm{PdO}(101)$. These are examples where the shifts calculated within a GC-functional deviate significantly from the measured values and where an admixture of Fock exchange improves the description. Moreover, as a reference, we study the so-called ESCA molecule, ethyl trifluoroacetate. Also in this case, the hybrid functionals yield shifts in better agreement with experiments. The improved agreement is analyzed in terms of the initial and final state contributions. 


\section{METHODOLOGY}

\section{A. Computational method}

The density functional theory is used as implemented in the VASP code. ${ }^{20-22}$ Calculations with a gradient-corrected functional are performed by use of the Perdew, Burke, and Ernzerhof (PBE) form ${ }^{23}$ and inclusion of Fock exchange is done by use of the PBE0 $0^{24,25}$ functional. In PBE0, $25 \%$ of the PBE exchange energy is replaced by Fock exchange.

Standard PBE Projector Augmented Wave (PAW) potentials ${ }^{26,27}$ are used to treat the interaction between the valence electrons and the core. The same PBE potentials are used in all calculations. The kinetic energy cut-off for the plane-wave basis set is set to $450 \mathrm{eV}$ which ensures adequate convergence for the considered potentials and elements. Reciprocal space integration over the Brillouin zone in the solids is approximated with finite sampling using Monkhorst-Pack grids $^{28,29}$ corresponding to $12 \times 12 \times 12$ in the unit cell of bulk Pd and $8 \times 8 \times 6$ in the unit cell of bulk PdO. Isolated molecules are treated in the $\Gamma$ point approximation. Geometry optimizations are carried out at the PBE level. This is done without any constraints, and the structures are considered relaxed when the largest force in the system is smaller than $0.01 \mathrm{eV} / \AA$. Periodically repeated images of isolated molecules and 2D slabs are separated by at least 12 A.

In order to estimate both initial and final state effects, two methods are used to evaluate the CLS. The initial contribution can be associated with the negative of the core level eigenvalue shift. ${ }^{30,31}$ This shift is, furthermore, closely related to changes in electrostatic potential at the nucleus. ${ }^{32,33}$ Here, the initial state contribution is estimated by probing differences in the electrostatic energy $\Delta V$ at the nucleus of the considered atoms.

The CLS including final state effects is calculated assuming complete screening of the core hole. In this method, the CLS is calculated as the total energy difference

$$
\mathrm{CLS}=\left[E_{*}-E_{0}\right]-\left[E_{*}^{\mathrm{ref}}-E_{0}^{\mathrm{ref}}\right] .
$$

Here, $E_{*}$ and $E_{0}$ represent the ground state energy of the system with and without a core hole, respectively. The complete screening approach assumes (i) that the lifetime of the core hole is long with respect to the photo-emission process, (ii) that the system is in the ground state with the constraint of a core hole, and (iii) that structural relaxations are negligible. These are approximations that are valid for the systems considered in the present study. ${ }^{3}$ The systems with a core hole are obtained by generating a PAW potential with an electron hole in either the $3 d$ shell $(\mathrm{Pd})$ or the $1 s$ shell $(\mathrm{C}$ and $\mathrm{O}) .{ }^{50}$ This approach is justified as the relaxation in the core region is not sensitive to the chemical environment.

As the periodic boundary conditions exclude the use of charged supercells, charge neutrality needs to be maintained in the presence of a core hole. This can be done either by adding an extra electron to the valence or by use of a compensating charge in the form of a homogeneous jellium background. Adding an extra electron is the preferred approach for metallic systems with a high density of states (DOS) at the Fermi level. On the other hand, for molecules (such the ESCA molecule), insulators, and semiconductors (such as $\mathrm{PdO}$ ), the jellium approach is more appropriate and is, consequently, used throughout the present study.

\section{B. Experimental method}

The HRCLS measurements were performed at beamline I311 at MAX IV Laboratory in Lund, Sweden. ${ }^{34}$ A normal emission angle was used and the $\mathrm{C} 1 s$ and $\mathrm{O} 1 \mathrm{~s}$ levels were recorded with photon energies of 435 and $650 \mathrm{eV}$, respectively. For the analysis of the recorded spectra, a deconvolution procedure involving a Doniach-Šunijć line shape together with a Gaussian is used. The sample preparation methods have previously been described in detail. ${ }^{35}$

\section{RESULTS AND DISCUSSION}

The primary interest of the present study is to evaluate the performance of DFT-based calculations to aid the interpretation of XPS measurements of surfaces with and without adsorbates. ${ }^{35-38}$ Here, we present experimental and computational results for $\mathrm{CO}$ adsorption on $\mathrm{PdO}(101)$. We will show that inclusion of Fock exchange enhances the agreement between the calculated and measured CLS. In order to put the results for $\mathrm{PdO}(101)$ surface in context, we also investigate the gas-phase ethyl trifluoroacetate molecule. This, so called, ESCA molecule ${ }^{39,40}$ has over the years been intensively studied and used as a reference. By the choice of systems, we are studying gas-phase molecules, molecules adsorbed on surfaces (CO on $\mathrm{PdO}(101))$ and solids (bulk Pd and PdO).

\section{A. The C $1 s$ shifts in the ESCA molecule}

The anti-anti rotamer of the ethyl trifluoroacetate molecule $\left(\mathrm{C}_{4} \mathrm{~F}_{3} \mathrm{H}_{5} \mathrm{O}_{2}\right)$ is shown as an inset of Figure 1. This rotamer is almost energetically degenerate with the antigauche structure and both isomers are experimentally observed in nearly equal amounts. ${ }^{41}$ The presented CLS are averages of the results for the two rotamers.

The previously reported experimental results are given in Table I. The lowest C $1 s$ binding energy of the four carbon atoms is measured for the methyl group (C1). The absolute binding energy is $291.2 \mathrm{eV}^{42}$ The binding energy of the methylene carbon atom (C2) is shifted to higher energies by $1.72 \mathrm{eV}$. The binding energies in the carboxylate (C3) and trifluoromethyl (C4) groups are shifted to even higher binding energies, namely, 4.33 and $7.46 \mathrm{eV}$, respectively. Within the PBE approximation, the calculated CLS with respect to the carbon atom in the methyl group are 1.64, 3.94, and $7.02 \mathrm{eV}$. Whereas the CLS for C2 is within $0.1 \mathrm{eV}$ of the experimental value, the shifts for $\mathrm{C} 3$ and $\mathrm{C} 4$ are underestimated by $\sim 0.4 \mathrm{eV}$. A clear improvement with respect to the experiments is obtained when the exchange-correlation instead is described within the PBE0 approximation. In this case, the discrepancy is within $0.15 \mathrm{eV}$ for all carbon atoms. This result is in agreement with a previous report using B3LYP (another hybrid functional) and an implementation of DFT with local basis functions. ${ }^{42}$

In order to understand the reason for the improved agreement, the initial state contribution is analyzed within the electrostatic $\Delta V$ model. The initial state contribution is in both 


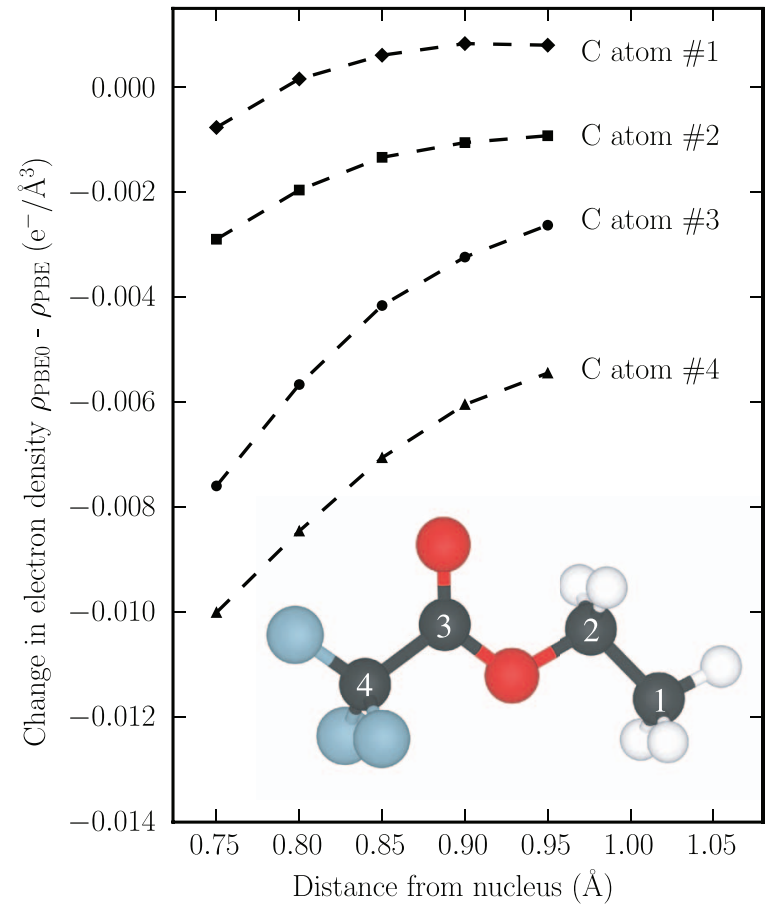

FIG. 1. Analysis of the valence charge density difference between PBE and PBE0 in the region outside the PAW sphere of the different carbon atoms in the anti-anti rotamer of the ESCA molecule.

functionals the major part of the CLS. A similar effect of Fock exchange is calculated for the $\Delta V$ results as for shifts calculated in the complete screening picture. This result suggests that the difference between PBE and PBE0 arises mainly from differences in the description of the initial state, i.e., differences in the valence charge density before ionization. In order to investigate this further, a Bader charge analysis was performed. ${ }^{43}$ The Bader charges on the carbon atoms within PBE were calculated to be 4.08, 3.70, 2.38, and 2.30 electrons for $\mathrm{C} 1-\mathrm{C} 4$, respectively. The corresponding values within PBE0 are 4.08, 3.69, 2.35, and 2.27 electrons. The differences are small but significant. This is demonstrated in the charge density difference analysis shown in Figure $1 .{ }^{51}$ Here, the difference in valence charge density between PBE and PBE0 is reported. The density difference is spherically averaged around each carbon nucleus and given as a function of the distance from the nucleus. Whereas all carbon atoms

TABLE I. Experimental C $1 s$ shifts for the ESCA molecule, together with the calculated CLS using different functionals in the complete screening picture and an electrostatic potential model. All theoretical shifts are averages of the anti-anti and anti-gauche rotamers.

\begin{tabular}{|c|c|c|c|c|c|c|}
\hline \multirow[b]{3}{*}{$\mathrm{C} \#$} & \multirow[b]{3}{*}{ Exp. $^{42}$} & \multicolumn{5}{|c|}{ C $1 s$ core level shift $(\mathrm{eV})$} \\
\hline & & \multicolumn{3}{|c|}{ Complete screening picture } & \multicolumn{2}{|c|}{ Potential model } \\
\hline & & B3LYP 42 & PBE0 & PBE & PBE0 & PBE \\
\hline 1 & 0 & 0 & 0 & 0 & 0 & 0 \\
\hline 2 & 1.72 & 1.74 & 1.76 & 1.64 & 1.67 & 1.56 \\
\hline 3 & 4.33 & 4.48 & 4.47 & 3.94 & 4.12 & 3.74 \\
\hline 4 & 7.46 & 7.50 & 7.57 & 7.02 & 6.50 & 6.01 \\
\hline
\end{tabular}

show a decrease in the valence charge density close to the nucleus upon inclusion of Fock exchange, $\mathrm{C} 3$ and $\mathrm{C} 4$ are most affected. Thus, Fock exchange enhances the charge separation in the $\mathrm{C}-\mathrm{O}$ and $\mathrm{C}-\mathrm{F}$ bonds, whereas the $\mathrm{C}-\mathrm{H}$ bonds are less affected. The increased ionicity in PBE0 as compared to PBE can be rationalized by the reduction of the self-interaction error which promotes charge localization. The general nature of this result was further investigated by calculating the CLS for diethyl carbonate. The $\mathrm{C} 1 s$ shift between the carbon atoms in the methyl and ester groups were calculated to be 4.27 and $4.84 \mathrm{eV}$ in PBE and PBE0, respectively.

We note that in the set of previously studied small $\mathrm{Ge}^{18}$ and $\mathrm{Si}^{11}$ containing molecules, the largest deviations with respect to experiments were calculated for molecules with multiple bonds to electronegative elements, such as in $\mathrm{GeF}_{4}$ $\left(\mathrm{Si}\left(\mathrm{OCH}_{3}\right)_{4}\right)$. This suggests that similar initial state effects occur for this class of molecules.

\section{B. The Pd 3d shift upon bulk oxidation of Pd}

High resolution core level spectroscopy has recently been used to measure the $\mathrm{Pd} 3 d$ binding energy in $\mathrm{PdO} .{ }^{35,44}$ The experiments were performed on oxidized Pd nanoparticles, and oxidized Pd single crystal surfaces. In both cases, the difference between the $3 d$ binding energy in the metal and the oxide was measured to be $1.6 \mathrm{eV}$. This CLS is clearly underestimated in PBE where it is calculated to be $\sim 1 \mathrm{eV}^{35,44,45}$

Here, we investigate the influence of Fock exchange on the Pd $3 d$ CLS between bulk palladium metal and bulk palladium oxide. As inclusion of both types of atoms in the same computational unit cell is advantageous to obtain accurate CLS, the calculations have been performed with a slab model consisting of commensurable $\mathrm{Pd}$ and $\mathrm{PdO}$ surfaces. In particular, the $\mathrm{PdO}(101)$ and $\mathrm{Pd}(100)$ surfaces are considered in the $(\sqrt{5} \times \sqrt{5}) R 27^{\circ} \mathrm{Pd}(100)$ surface cell. ${ }^{2}$ With respect to $\mathrm{PdO}(101)$, this corresponds to a slightly compressed (1.4\% in surface area) $(2 \times 1)$ surface cell. The model, which is shown in Figure 2, consists of three layers of $\operatorname{Pd}(100)$ and three trilayers of $\mathrm{PdO}(101)$. That this model is sufficient to converge the CLS with respect to the number of layers was confirmed by PBE calculations with seven metal layers and seven trilayers of the oxide. The Pd $3 d$ CLS for the small model is calculated to be $0.90 \mathrm{eV}$, whereas the CLS for the large model is $0.91 \mathrm{eV}$.

The PBE and PBE0 results are compared in Table II. Within the complete screening method, the Pd $3 d$ CLS is calculated to be $2.00 \mathrm{eV}$, which, although an overestimation, is in better agreement with the experiments. It should be noted that the magnitude of the shift in PBE0 depends on the amount of Fock exchange that is used and that the optimal mixing is system and property dependent. ${ }^{24,52}$

As in the case of the ESCA molecule, the charge separation is slightly larger in PBE0 as compared to PBE. A Bader charge analysis gives that the metal charge in bulk $\mathrm{PdO}$ is 9.17 $\mathrm{e}^{-}$in PBE, whereas it is $9.11 \mathrm{e}^{-}$in PBE0. As the valence charge of the CLS reference atom (Pd in the metal) is unaffected by the functional, this suggests that a part of the CLS change can be attributed to initial state effects. This is consistent with the results from $\Delta V$ calculations which are shown 


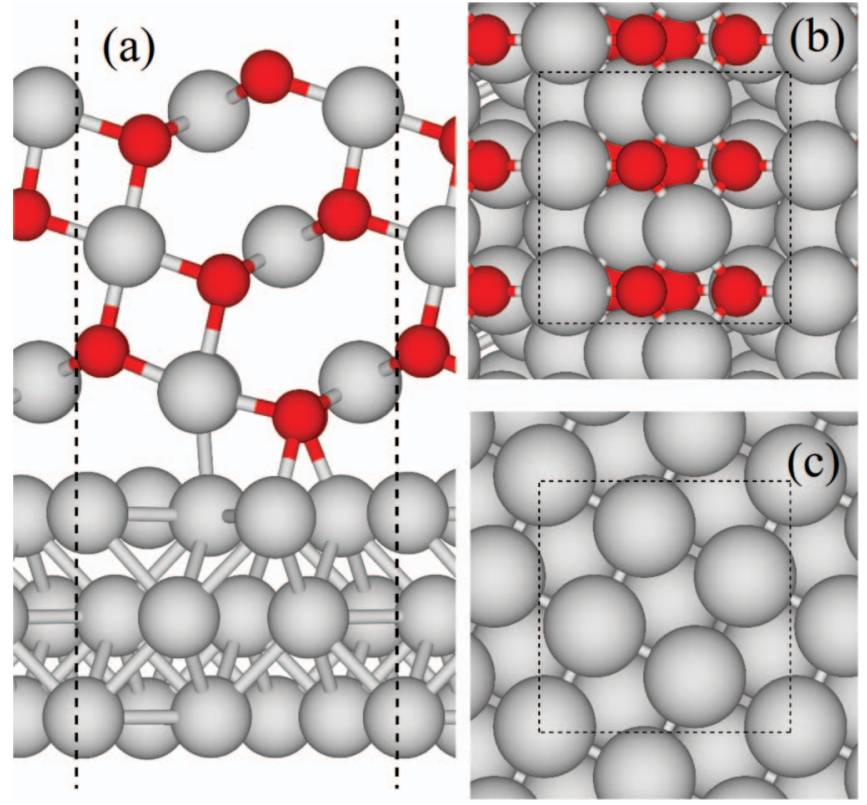

FIG. 2. Side (a) and top (b) and (c) views of the $\operatorname{PdO}(101)$ overlayer on $\operatorname{Pd}(100)$. The unit cell is drawn as a dashed black line.

in Table II. The initial state contribution is in both functionals a large part of the total CLS. In PBE, the final state effects amounts to $0.20 \mathrm{eV}$, whereas it is $0.67 \mathrm{eV}$ in PBE0. This result is slightly different from the ESCA molecule where the final state effects were calculated to be similar for PBE and $\mathrm{PBE}$. This indicates that the band gap that develops for $\mathrm{PdO}$ in PBE0 also affects the results.

In order to understand the final state effects, the systems with the core hole were investigated in detail. A Bader charge analysis of the valence charge distribution shows that in both the metal and oxide, the core excited Pd atom gains close to one valence electron as a result of electronic screening. This result is valid for both functionals and indicates that a deeper analysis of the screening process should be performed. We do this by investigating the electronic DOS.

As the screening charge on the $\mathrm{Pd}$ atoms will be mainly of $d$ character, we focus on the Pd $4 d$ states. In Figure 3 , the projected density of states (PDOS) on a bulk atom before and after core excitation is shown. For the metal (bottom panels), the initial PDOS shows the almost filled $d$-band that cuts the Fermi energy, whereas the final state PDOS reveal a completely filled local $d$-band. The two functionals are providing similar results. We note that the width of the $d$-band is larger (and actually overestimated) in PBE0. ${ }^{46}$

TABLE II. Calculated Pd $3 d$ CLS (eV) in the complete screening picture and the electrostatic potential model using the PBE and PBE0 functionals.

\begin{tabular}{llllll}
\hline \hline & \multicolumn{2}{c}{$\begin{array}{c}\text { Complete screening } \\
\text { picture }\end{array}$} & & \multicolumn{2}{c}{$\begin{array}{c}\text { Potential } \\
\text { model }\end{array}$} \\
\cline { 2 - 3 } \cline { 5 - 6 } Pd atom & PBE & PBE0 & & PBE & PBE0 \\
\hline Pd (bulk) & 0 & 0 & & 0 & 0 \\
PdO (bulk) & 0.90 & 2.00 & & 0.70 & 1.33 \\
\hline \hline
\end{tabular}
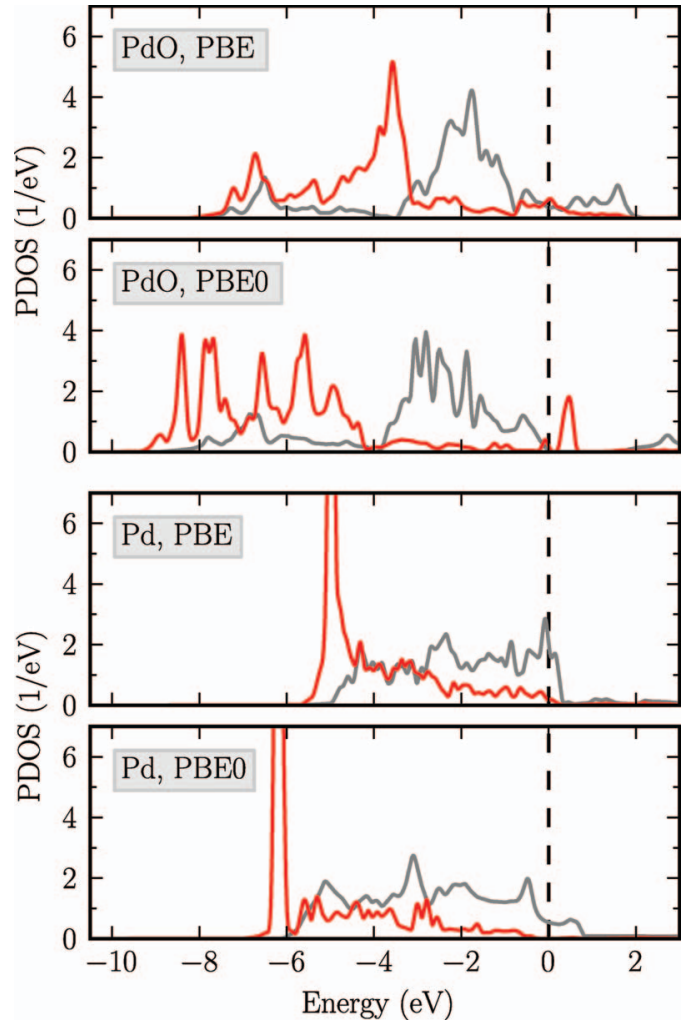

FIG. 3. Electronic DOS projected on the $d$-states of a bulk Pd atom in Pd and $\mathrm{PdO}$ with the PBE or PBE0 functionals, both before (gray) and after (red) creation of a core hole. The one-electron Kohn-Sham energies are broadened with a $0.3 \mathrm{eV}$ Gaussian. The PDOS is for PdO reported with respect to the highest occupied level.

Turning to PdO (top panels), the occupied PDOS in the initial state is similar for the two functionals. One important difference is instead the band gap that appears when Fock exchange is included. ${ }^{45}$ In PBE0, we calculate a Kohn-Sham band gap of $1.7 \mathrm{eV}$. Experimentally, the band gap of $\mathrm{PdO}$ has been determined to be $0.8 \mathrm{eV}$ (optical ${ }^{47}$ ) and $1.5 \mathrm{eV}$ $\left(\right.$ conductivity ${ }^{48}$ ), respectively. Creation of a core hole in $\mathrm{PdO}$ again leads to stabilisation of the occupied states. Screening of the core hole results in occupation of $d$ states that initially are unoccupied. Because the sum of Kohn-Sham eigenvalues constitutes a large part of the total electronic energy, it is clear that the electronic relaxation energy in the oxide, as compared to the metal, will be reduced in PBE0 owing to the band gap.

The present calculations are performed by use of a metal/metal-oxide interface. In principle, this could affect the screening in the oxide by access of mobile electrons from the metal. However, the core hole screening in the investigated oxide is metal-like in the sense that many atoms contribute equally to the screening charge. Thus, the presence of the Pd metal substrate will not affect the core hole screening significantly. The situation is rather different for the ESCA molecule where the screening charge mainly originates from the nearest neighbour atoms. Note that the $\mathrm{Pd} 3 d$ CLS calculated for Pd(111) with PBE and PBE0 are similar, -0.30 and $-0.30 \mathrm{eV}$, respectively. This is in agreement with the common experience that PBE is suitable for pure metal systems. 


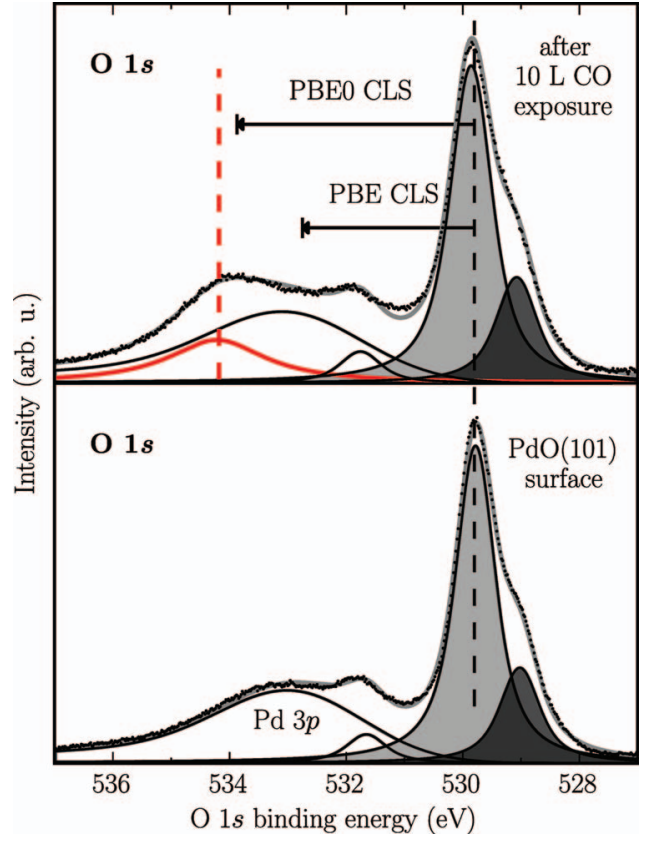

FIG. 4. Measured O $1 s$ binding energies for the clean and CO-covered $\mathrm{PdO}(101)$ surface, together with theoretical shifts calculated with the PBE and PBE0 functionals. Aside from a Pd $3 p_{3 / 2}$ peak and a surface hydroxyl contamination peak, the deconvolutions are: bulk-like fourfold coordinated oxygen atoms (gray), threefold coordinated surface oxygen atoms (black), and oxygen in adsorbed $\mathrm{CO}$ (red).

\section{The $\mathrm{O} 1 \mathrm{~s}$ shift of $\mathrm{CO}$ adsorbed on $\mathrm{PdO}(101)$}

The experimental results for the $\mathrm{O} 1 s$ spectra after $\mathrm{CO}$ adsorption on $\mathrm{PdO}(101)$ at $100 \mathrm{~K}$ are shown in Figure 4. The bottom panel shows the spectrum obtained from the bare $\mathrm{PdO}(101)$ surface, whereas the top panel is the result after CO exposure. The $\mathrm{O} 1 s$ spectra are somewhat obscured by the $\mathrm{Pd}$ $3 p_{3 / 2}$ peak that appears in the same energy range. The spectrum for the bare surface is deconvoluted into three peaks. The large peak at $529.8 \mathrm{eV}$ is assigned to $\mathrm{O}$ atoms in bulk $\mathrm{PdO}$, whereas the peak at $529.0 \mathrm{eV}$ is assigned to $\mathrm{O}$ atoms at the surface. The small peak at $531.6 \mathrm{eV}$ has previously been attributed to hydroxyl groups. ${ }^{38}$ Upon $\mathrm{CO}$ exposure, a new feature appears at $534.1 \mathrm{eV}$ which is attributed to adsorbed $\mathrm{CO}$. This peak is shifted by $4.3 \mathrm{eV}$ with respect to $\mathrm{O} 1 \mathrm{~s}$ in the PdO bulk.

$\mathrm{CO}$ can adsorb in either an atop or a bridge configuration on $\mathrm{PdO}(101)$. At a coverage of $0.5 \mathrm{ML}$ (with respect to threefold Pd sites), the two sites are within PBE calculated to be energetically degenerate with an adsorption energy of $1.46 \mathrm{eV}^{53}$ For a five trilayer $\mathrm{PdO}(101)$ slab, the O $1 s$ CLS within PBE of $\mathrm{CO}$ with respect to $\mathrm{PdO}$ bulk is $1.16 \mathrm{eV}$ and $2.95 \mathrm{eV}$ for the bridge and atop configuration, respectively. ${ }^{54}$ A comparison between the calculations and the experiments in Figure 4 suggests that the bridge $\mathrm{CO}$ signal is masked by the $\mathrm{Pd} 3 p_{3 / 2}$ and hydroxyl components and that the peak at $534.1 \mathrm{eV}$ originates from atop CO. Applying the PBE0 functional to atop CO results in a CLS of $4.07 \mathrm{eV}$ which is close to the experimental value. Also the CLS for bridge $\mathrm{CO}$ is increased by $1 \mathrm{eV}$ to $2.10 \mathrm{eV}$.

Using the $\Delta V$ model, we estimate the initial state contribution to the CLS of atop $\mathrm{CO}$ to be $2.26 \mathrm{eV}$ and $3.21 \mathrm{eV}$ in
PBE and PBE0, respectively. Thus, the contribution in the final state CLS from electronic screening amounts in both cases to about $0.8 \mathrm{eV}$ and the difference between the complete PBE and PBE0 shifts can be understood in terms of initial state effects. The Bader charges for oxygen in bulk PdO are $6.79 \mathrm{e}^{-}$ (PBE) and $6.85 \mathrm{e}^{-}$(PBE0). The corresponding values for oxygen in adsorbed $\mathrm{CO}$ are $7.01 \mathrm{e}^{-}$(PBE) and $7.03 \mathrm{e}^{-}$(PBE0). Whereas the Bader charge associated with the oxygen atom in the adsorbate is very close in PBE and PBE0, the atomic charge of the oxygen atom in the bulk of the oxide is 0.06 $\mathrm{e}^{-}$higher in PBE0 as compared to PBE. This is a situation that is similar to the ESCA molecule and the Pd $3 d$ CLS for bulk $\mathrm{PdO}$, where the inclusion of Fock exchange is found to promote the charge separation.

In a previous study, ${ }^{19}$ the $\mathrm{O} 1 \mathrm{~s}$ core level shifts of water adsorbed on $\mathrm{MgO}(100)$ were investigated with both $\mathrm{PBE}$ and PBE0. In this case, the two functionals predict similar CLS. To understand this, we reproduced the calculations for a $(3 \times 2)$ partially dissociated $\mathrm{H}_{2} \mathrm{O}$ monolayer on $\mathrm{MgO}(100)$ with our computational setup. The CLS of interest are those of adsorbed $\mathrm{H}_{2} \mathrm{O}$, adsorbed $\mathrm{OH}$, surface $\mathrm{O}$, and surface $\mathrm{OH}$ with respect to $\mathrm{O}$ in the bulk of the $\mathrm{MgO}$. For these species, we find that the initial state contribution makes up the majority of the CLS and that differences in the initial state contribution between PBE and PBE0 only amounts to about $0.1 \mathrm{eV}$. A Bader analysis of the valence charge density shows that the $\mathrm{O}$ atoms in the different species consistently gain 0.01-0.02 electrons in PBE0 as compared to PBE. Hence, the differences in charge separation are not altered by inclusion of Fock exchange for this system and clarifies why the differences in the initial state contribution between PBE and PBE0 are small. Furthermore, the system has a large band-gap $(\sim 2.7 \mathrm{eV}$ in PBE) which prevents the occupation of previously empty states during the screening process. This provides an explanation why the final state contributions to the CLS are not much influenced by the inclusion of Fock exchange.

\section{CONCLUSIONS}

Density functional theory calculations with gradientcorrected exchange-correlation functionals have repeatedly been demonstrated to reproduce experimental CLS with reasonable accuracy. Here, we have investigated examples that illustrate limitations of such functionals with the aim to identify cases where usage of higher-level functionals is desirable. As XPS is sensitive to charge transfers, the exchange of electrons has to be well described in order to accurately determine the CLS without relying too much on a cancellation of errors. With gradient-corrected functionals, the self-interaction error can lead to an underestimation of charge transfer in polar bonds, which yield too small CLS. In the current work, this is illustrated by calculations for the ESCA molecule and CO adsorbed on the $\mathrm{PdO}(101)$ surface. In solid systems, screening of the core hole can entail occupation of previously empty states. Thus, the general underestimation of band gaps within gradient-corrected functionals can then lead to an underestimation of the final state contribution, as shown in the present work by comparing the CLS of bulk palladium oxide and bulk palladium. 


\section{ACKNOWLEDGMENTS}

The Max IV staff is gratefully acknowledged. Financial support is acknowledged from the Swedish Research Council, Chalmers Areas of Advance Nano and Transport, the Crafoord Foundation, the Knut and Alice Wallenberg Foundation, the Anna and Edwin Berger Foundation, NordForsk and COST action CM1104. The calculations were performed at C3SE (Göteborg) via a SNIC grant. The Competence Centre for Catalysis (KCK) is hosted by Chalmers University of Technology and is financially supported by the Swedish Energy Agency and the member companies: AB Volvo, ECAPS AB, Haldor Topsøe A/S, Scania CV AB, Volvo Car Corporation AB, and Wärtsilä Finland Oy. J.F.W. gratefully acknowledges financial support by the (U.S.) Department of Energy (DOE), Office of Basic Energy Sciences, Catalysis Science Division through Grant No. DEFG02-03ER15478.

${ }^{1}$ E. Lundgren, G. Kresse, C. Klein, M. Borg, J. N. Andersen, M. De Santis, Y. Gauthier, C. Konvicka, M. Schmid, and P. Varga, Phys. Rev. Lett. 88, 246103 (2002).

${ }^{2}$ M. Todorova, E. Lundgren, V. Blum, A. Mikkelsen, S. Gray, J. Gustafson, M. Borg, J. Rogal, K. Reuter, J. N. Andersen, and M. Scheffler, Surf. Sci. 541, 101 (2003).

${ }^{3}$ J. N. Andersen and C.-O. Almbladh, J. Phys.: Condens. Matter 13, 11267 (2001).

${ }^{4}$ M. Birgersson, C.-O. Almbladh, M. Borg, and J. N. Andersen, Phys. Rev. B 67, 045402 (2003).

${ }^{5}$ O. Takahashi and L. G. M. Pettersson, J. Chem. Phys. 121, 10339 (2004).

${ }^{6}$ A. Alkauskas, P. Broqvist, and A. Pasquarello, Phys. Status Solidi B 248, 775-789 (2011).

${ }^{7}$ A. Alkauskas and A. Pasquarello, Phys. Rev. B 84, 125206 (2011).

${ }^{8}$ N. Mårtensson and A. Nilsson, J. Electron Spectrosc. Relat. Phenom. 75, 209 (1995).

${ }^{9}$ E. Pehlke and M. Scheffler, Phys. Rev. Lett. 71, 2338 (1993).

${ }^{10}$ J. N. Andersen, D. Hennig, E. Lundgren, M. Methfessel, R. Nyholm, and M. Scheffler, Phys. Rev. B 50, 17525 (1994).

${ }^{11}$ A. Pasquarello, M. S. Hybertsen, G.-M. Rignanese, and R. Car, in Fundamental Aspects of Ultrathin Dielectrics on Si-based Devices, NATO Science Series Vol. 47, edited by E. Garfunkel, E. Gusev, and A. Vul (Springer, Netherlands, 1998), pp. 89-102.

${ }^{12}$ S. Lizzit, A. Baraldi, A. Groso, K. Reuter, M. V. Ganduglia-Pirovano, C. Stampfl, M. Scheffler, M. Stichler, C. Keller, W. Wurth, and D. Menzel, Phys. Rev. B 63, 205419 (2001).

${ }^{13}$ L. Köhler and G. Kresse, Phys. Rev. B 70, 165405 (2004).

${ }^{14}$ J. G. Wang, W. X. Li, M. Borg, J. Gustafson, A. Mikkelsen, T. M. Pedersen, E. Lundgren, J. Weissenrieder, J. Klikovits, M. Schmid, B. Hammer, and J. N. Andersen, Phys. Rev. Lett. 95, 256102 (2005).

${ }^{15}$ W. Olovsson, C. Göransson, T. Marten, and I. A. Abrikosov, Phys. Status Solidi B 243, 2447-2464 (2006).

${ }^{16}$ J. Jaramillo, G. E. Scuseria, and M. Ernzerhof, J. Chem. Phys. 118, 1068 (2003).

${ }^{17}$ S. L. Dudarev, G. A. Botton, S. Y. Savrasov, C. J. Humphreys, and A. P. Sutton, Phys. Rev. B 57, 1505 (1998).

${ }^{18}$ J. F. Binder, P. Broqvist, H.-P. Komsa, and A. Pasquarello, Phys. Rev. B 85, 245305 (2012)

${ }^{19}$ L. O. Paz-Borbón, A. Hellman, and H. Grönbeck, J. Phys. Chem. C 116, 3545 (2012).

${ }^{20}$ G. Kresse and J. Furthmüller, Comput. Mater. Sci. 6, 15 (1996).

${ }^{21}$ G. Kresse and J. Furthmüller, Phys. Rev. B 54, 11169 (1996).

${ }^{22}$ G. Kresse and J. Hafner, Phys. Rev. B 49, 14251 (1994).

${ }^{23}$ J. P. Perdew, K. Burke, and M. Ernzerhof, Phys. Rev. Lett. 77, 3865 (1996).

${ }^{24}$ J. P. Perdew, M. Ernzerhof, and K. Burke, J. Chem. Phys. 105, 9982 (1996).

${ }^{25}$ C. Adamo and V. Barone, J. Chem. Phys. 110, 6158 (1999).
${ }^{26}$ P. E. Blöchl, Phys. Rev. B 50, 17953 (1994).

${ }^{27}$ G. Kresse and D. Joubert, Phys. Rev. B 59, 1758 (1999).

${ }^{28}$ H. J. Monkhorst and J. D. Pack, Phys. Rev. B 13, 5188 (1976).

${ }^{29}$ J. D. Pack and H. J. Monkhorst, Phys. Rev. B 16, 1748 (1977).

${ }^{30}$ A. R. Williams and N. D. Lang, Phys. Rev. Lett. 40, 954 (1978).

${ }^{31}$ D. Spanjaard, C. Guillot, M.-C. DesjonquÃ “res, G. Tréglia, and J. Lecante, Surf. Sci. Rep. 5, 1 (1985).

${ }^{32}$ C. Nordling, S. Hagström, and K. Siegbahn, Z. Phys. 178, 433 (1964).

${ }^{33}$ P. S. Bagus, E. S. Ilton, and C. J. Nelin, Surf. Sci. Rep. 68, 273 (2013).

${ }^{34}$ R. Nyholm, J. N. Andersen, U. Johansson, B. N. Jensen, and I. Lindau, Nucl. Instrum. Methods Phys. Res. A 467-468, 520 (2001).

${ }^{35}$ N. M. Martin, M. Van den Bossche, H. Grönbeck, C. Hakanoglu, F. Zhang, T. Li, J. Gustafson, J. F. Weaver, and E. Lundgren, J. Phys. Chem. C 118, 1118 (2014).

${ }^{36}$ J. Knudsen, N. M. Martin, E. Grånäs, S. Blomberg, J. Gustafson, J. N. Andersen, E. Lundgren, S. Klacar, A. Hellman, and H. Grönbeck, Phys. Rev. B 84, 115430 (2011).

${ }^{37}$ S. Klacar, N. M. Martin, J. Gustafson, S. Blomberg, Z. Liu, S. Axnanda, R. Chang, E. Lundgren, and H. Grönbeck, Surf. Sci. 617, 167 (2013).

${ }^{38}$ N. M. Martin, M. Van den Bossche, H. Grönbeck, C. Hakanoglu, J. Gustafson, S. Blomberg, M. A. Arman, A. Antony, R. Rai, A. Asthagiri, J. F. Weaver, and E. Lundgren, J. Phys. Chem. C 117, 13510 (2013).

${ }^{39}$ K. Siegbahn and C. Nordling, ESCA, Atomic, Molecular and Solid State Structure Studied by Means of Electron Spectroscopy (Almqvist \& Wiksell, 1967).

${ }^{40}$ K. Siegbahn, ESCA Applied to Free Molecules (North-Holland Pub. Co., 1970).

${ }^{41}$ M. E. Defonsi Lestard, M. E. Tuttolomondo, D. A. Wann, H. E. Robertson, D. W. H. Rankin, and A. B. Altabef, J. Raman Spectrosc. 41, 1357-1368 (2010).

${ }^{42}$ O. Travnikova, K. J. Børve, M. Patanen, J. Söderström, C. Miron, L. J. Sæthre, N. Mårtensson, and S. Svensson, J. Electron Spectrosc. Relat. Phenom. 185, 191 (2012).

${ }^{43}$ R. F. W. Bader, Encyclopedia of Computational Chemistry (John Wiley and Sons, Ltd., 2002).

${ }^{44}$ R. Westerström, M. E. Messing, S. Blomberg, A. Hellman, H. Grönbeck, J. Gustafson, N. M. Martin, O. Balmes, R. van Rijn, J. N. Andersen, K. Deppert, H. Bluhm, Z. Liu, M. E. Grass, M. Hävecker, and E. Lundgren, Phys. Rev. B 83, 115440 (2011).

${ }^{45}$ N. Seriani, J. Harl, F. Mittendorfer, and G. Kresse, J. Chem. Phys. 131, 054701 (2009).

${ }^{46}$ J. Paier, M. Marsman, K. Hummer, G. Kresse, I. C. Gerber, and J. G. Ángyán, J. Chem. Phys. 124, 154709 (2006).

${ }^{47}$ P. O. Nilsson, J. Phys. C 12, 1423 (1979).

${ }^{48}$ H. Okamoto and T. Asô, Jpn. J. Appl. Phys. 6, 779 (1967).

${ }^{49}$ H. Grönbeck and M. Odelius, Phys. Rev. B 82, 085416 (2010).

${ }^{50}$ In VASP, the core-excited PAW potential can be automatically generated from the provided PAW potential by removing the desired core electron. The other core electrons are in this approach kept frozen, which is justified as screening of the core hole by the other core electrons has little influence on the core-level shift. ${ }^{4,18}$

${ }^{51}$ Also the second moments of the valence charge density distribution close to the carbon nuclei were computed, in order to compare the degree of charge localization for the different functionals. The values of the second moments calculated with PBE (PBE0) are 0.513 (0.515), 0.525 (0.527), $0.544(0.546)$, and $0.569 \AA^{2}\left(0.571 \AA^{2}\right)$ for carbon atoms 1 till 4 , respectively. The second moments are very similar in both functionals, which indicates that charge transfer, rather than charge localization, is the main result upon inclusion of the exact exchange.

${ }^{52}$ The experimental CLS of $1.6 \mathrm{eV}$ is obtained with an admixture of $16 \%$ of Fock exchange.

${ }^{53}$ The binding energies within PBE0 are $1.38 \mathrm{eV}$ and $0.60 \mathrm{eV}$ for the atop and bridge positions, respectively.

${ }^{54}$ When the lateral dimension of the $\mathrm{PdO}(101)$ surface cell is increased to $(4 \times 2)$ and $(6 \times 3)$, the $\mathrm{O} 1 s$ shift for $\mathrm{CO}$ adsorbed atop a threefold coordinated $\mathrm{Pd}$ atom, is increased to $3.19 \mathrm{eV}$ and $3.23 \mathrm{eV}$. Thus, this particular CLS has a dependence on the concentration of core excited molecules. Note that the dependence on surface cell dimension does not affect the comparison between PBE and PBE0. 